\title{
Active Vibration Suppression of a 3-DOF Flexible Parallel Manipulator Using Efficient Modal Control
}

\author{
Quan Zhang, Jiamei Jin, Jianhui Zhang, and Chunsheng Zhao \\ State Key Laboratory of Mechanics and Control of Mechanical Structures, Nanjing University of Aeronautics and Astronautics, \\ Nanjing 210016, China
}

Correspondence should be addressed to Quan Zhang; quanzhang@nuaa.edu.cn

Received 1 May 2014; Accepted 20 June 2014; Published 20 October 2014

Academic Editor: Jeong-Hoi Koo

Copyright (C) 2014 Quan Zhang et al. This is an open access article distributed under the Creative Commons Attribution License, which permits unrestricted use, distribution, and reproduction in any medium, provided the original work is properly cited.

This paper addresses the dynamic modeling and efficient modal control of a planar parallel manipulator (PPM) with three flexible linkages actuated by linear ultrasonic motors (LUSM). To achieve active vibration control, multiple lead zirconate titanate (PZT) transducers are mounted on the flexible links as vibration sensors and actuators. Based on Lagrange's equations, the dynamic model of the flexible links is derived with the dynamics of PZT actuators incorporated. Using the assumed mode method (AMM), the elastic motion of the flexible links are discretized under the assumptions of pinned-free boundary conditions, and the assumed mode shapes are validated through experimental modal test. Efficient modal control (EMC), in which the feedback forces in different modes are determined according to the vibration amplitude or energy of their own, is employed to control the PZT actuators to realize active vibration suppression. Modal filters are developed to extract the modal displacements and velocities from the vibration sensors. Numerical simulation and vibration control experiments are conducted to verify the proposed dynamic model and controller. The results show that the EMC method has the capability of suppressing multimode vibration simultaneously, and both the structural and residual vibrations of the flexible links are effectively suppressed using EMC approach.

\section{Introduction}

With increasing demands of high speed and lightweight manipulators, robots with flexible links are designed and applied in many industrial fields, such as semiconductor manufacturing, astronautics, and automatic microassembly. Compared with traditional rigid manipulator, such robots have the advantages of high speed and acceleration, lower energy consumptions, and greater payload-to-arm weight ratio [1]. However, the vibrations will be introduced in the system due to the flexibility, leading to longer settling time and lower motion tracking accuracy of the end-effector. Hence, vibration control technologies play a critical role in the field of flexible robots. Compared with conventional passive vibration control approaches, active vibration control can suppress multiple vibration modes simultaneously, has the ability to adapt the system and environmental variations, and usually is more effective than passive method [2]. During active vibration control process, the vibration actuators bonded on the flexible parts generate forces according to the feedback signals of the displacement or velocity measured by the vibration sensors. If the output voltages of the vibration sensors are amplified properly through the active vibration controller, the actuator forces will increase the stiffness and damping of the flexible parts, thus attenuating the amplitude of the unwanted vibrations. With the development of the PZT materials, more and more flexible structures are mounted with multiple PZT transducers to achieve self-sensing and active vibration control $[3,4]$. The dynamic modeling and control of these smart manipulators have been investigated by many researchers. Early investigations have focused primarily on modeling of serial flexible space arms or single flexible beams [5-7], and a detailed review was provided by Dwivedy and Eberhard [8]. In contrast, little research literature on vibration suppression of parallel robot with smart flexible links has been published. Wang and Mills [9] presented a finite element model (FEM) of a PPM with elastic linkages for active vibration analysis using substructure methodology. Piras et al. [10] analyzed the dynamics of a planar fully parallel robot with flexible links using FEM. Zhang et al. [11] 
formulated a dynamic model of a PPM based on pinnedpinned boundary conditions based on the assumed mode method. Yu et al. [12] conducted both theoretical and experimental studies on the dynamic analysis of a 3-RRR flexible parallel robot. However, the dynamic modeling of flexible parallel manipulator is a challenging work due to its complex coupling characteristics between the rigid motion and elastic deformation.

Based on the dynamic model and vibration transducers, a variety of active vibration control approaches have been studied since the 1970s. In [13]; two different vibration control laws, namely, velocity feedback control and positive position feedback control (PPF), are studied based on a single flexible beam mounted with single pair of PZT transducer. Zhang et al. [14] conducted strain rate feedback control to suppress unwanted oscillation of a 3-PRR flexible parallel manipulator with smart flexible links. Combined with input shaper and multimode PPF controller, Chu et al. [15] suppressed the residual vibration of a high speed flexible manipulator. Considering the exact boundary conditions, Zhang et al. [16] achieved active vibration control of a flexible parallel robot using input shaping control approach. To achieve high accuracy trajectory tracking while attenuating the structural vibration simultaneously, Zhang et al. [17] implemented variable structural control (VSC) and direct output feedback control (strain and strain rate feedback) on a flexible parallel manipulator driven by LUSM. However, usually only a limited small number of modes are selected to be controlled in practice, and hence the uncontrolled modes may lead to spillover, a phenomenon in which controls energy flows to the uncontrolled modes of the system [18]. To prevent the recoupling of modal equations through feedback and spillover, Meirovitch and Baruh [19] proposed the independent modal space control (IMSC) approach. In this method, the control problem of continuous structure can be understood as controlling several single degree of freedom systems simultaneously, and each mode of the continuous structure is controlled by one independent controller related to its own modal displacement and velocity. However, the feedback gains of IMSC are calculated off-line and the control voltage for high modes is relatively high. When some vibration modes are excited to a greater extent by the disturbances during the operation, neither priority nor adjustment of feedback gains is given to these excited modes. Based on IMSC, $\mathrm{Baz}$ and Poh [20] developed modified independent modal space control (MIMSC) to overcome these disadvantages through controlling the different vibration modes depending upon its own energy. The vibration energy of each mode is identified at every intervals and the mode with highest vibration energy is controlled first. Although the different modes are prioritized and the applied voltages are decreased when using MIMSC, it will put computational efforts on the digital controller since the MIMSC needs to weigh and compare the vibration energies of all modes at every intervals. Singh et al. [21] proposed an efficient modal control (EMC) method to suppress multiple vibration modes of a cantilever beam. Compared with IMSC and MIMSC, the EMC method has a simpler feedback gains and lower amplitude of control voltages, which can be readily implemented on a controller.
Hence the EMC method is adopted to suppress the vibration of the flexible link in this study.

This paper addresses the dynamic modeling and active vibration control of a PPM with three smart flexible links. Firstly, the dynamic model of the flexible links mounted with multiple PZT transducers is formulated using Lagrange's equations and AMM, and the experimental modal tests of the flexible links are implemented to verify the assumed mode shapes. Then, based on the presented dynamic model, the efficient modal control strategies are adopted to realize the active vibration control of the flexible links through multiple PZT transducers. Finally, MATLAB simulations and vibration control experiments are provided to validate the effectiveness of the proposed control approach.

\section{Dynamic Model of the Flexible Links Incorporated with PZT Transducers}

2.1. Introduction of the Flexible Parallel Manipulator. Considering the beneficial characteristics of parallel structure manipulator with flexible links, a 3-PRR-type PPM with light links driving by LUSMs is developed to achieve planar motion tracking and positioning task, that is, with coordinates of $x-y-\varphi$. The prototype and coordinates system of the PPM are shown in Figure 1 . Variables $\alpha_{i}$ and $\beta_{i}(i=1,2,3)$ represent the layout angle of the three linear guides and the angle between the $x$-axis of the static frame and $i$ th links, respectively. $L_{i}$ is the length of the linkage. The prismatic motion of the LUSM is defined as $\rho_{i}$. The coordinates of the moving platform are represented as a vector of $\bar{X}_{p}=$ $\left(x_{p}, y_{p}, \varphi_{p}\right)^{T}$ in the static coordinate system, and $w_{i}\left(x_{i}\right)$ is the elastic deformation at the $x_{i}\left(0 \leq x_{i} \leq L_{i}\right)$ of the $i$ th linkage. The detailed parameter definitions are shown in our preliminary study [17].

2.2. Discretization of the Elastic Motion. The elastic motions $w_{i}$ in Figure 1 need to be discretized first for further dynamic analysis. Since the length of the flexible link is much longer than its thickness, the Euler-Bernoulli beam theory is adopted to model the flexible link, and hence only the transverse vibration of the link is considered. According to AMM, the elastic motion of the $i$ th flexible links can be presented as

$$
w_{i}(x, t)=\sum_{j=1}^{r} Y_{i j}(x) q_{i j}(t), \quad i=1,2,3,
$$

where $q_{i j}(t)$ represents the unknown generalized coordinates of the $j$ th mode of the $i$ th link and $Y_{i j}(x)$ are the spatial shape functions.

Literatures [11, 16] show that the moving platform will vibrate fiercely due to the deformation of the flexible link in such rigid-flexible PPM, especially under the operation of high speed and high acceleration. Hence we may consider the beginning of the flexible link is pinned at point $B_{i}$ while the end of the link is free but modeled with constraint forces applied by joint $C_{i}$. Hence the normalized shape functions 


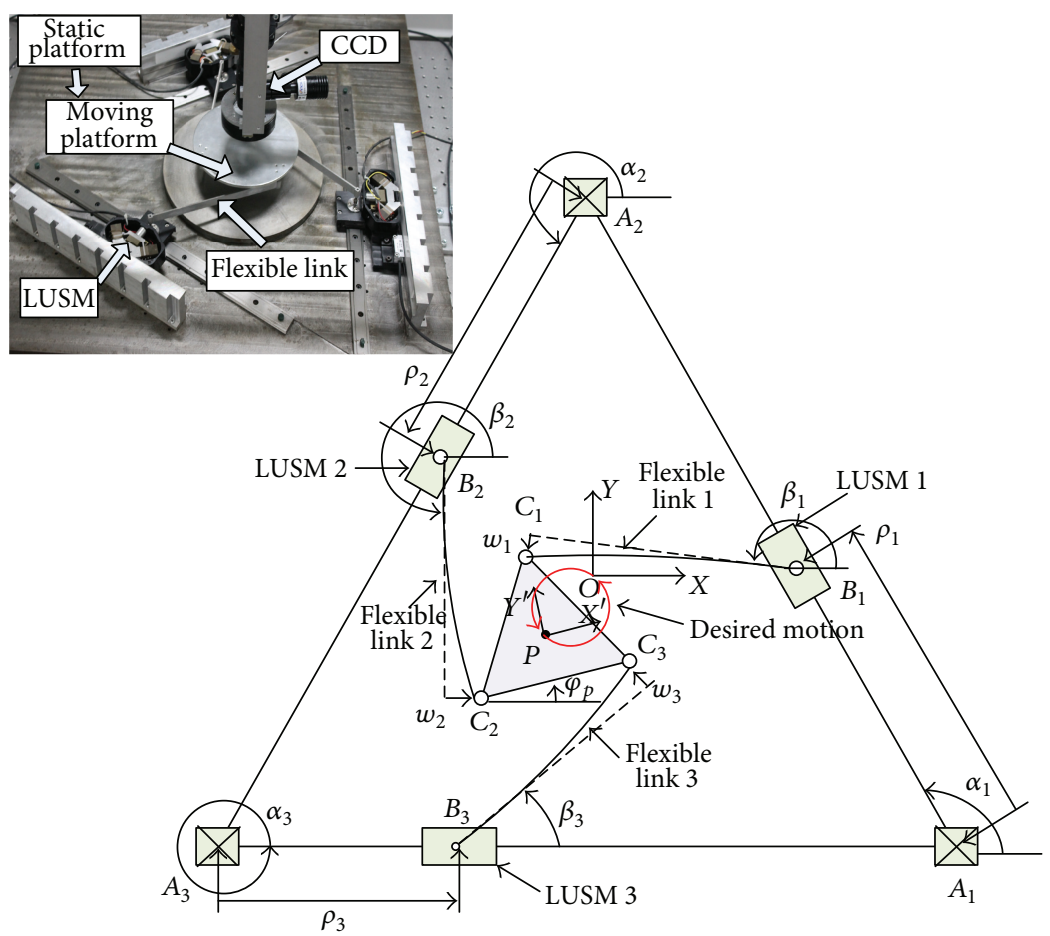

FIgURe 1: Prototype and schematic model of the PPM.

matching pinned-free boundary conditions are adopted to model the flexible links as follows [22]:

$$
\begin{aligned}
& Y_{i j}(x) \\
& =\frac{1}{\sqrt{m_{i} L_{i}} \sin \theta_{j}}\left[\sin \left(\frac{\theta_{j} x_{i}}{L_{i}}\right)+\frac{\sin \theta_{j}}{\sinh \theta_{j}} \sinh \left(\frac{\theta_{j} x_{i}}{L_{i}}\right)\right],
\end{aligned}
$$

where $\theta_{j}=(j+0.25) \pi, j=1,2, \ldots, r$, and $0 \leq x_{i} \leq L_{i}$.

2.3. Modal Testing Experiment. To validate the assumptions of pinned-free mode shapes used in the dynamic model, experimental modal tests are carried out. As shown in Figure 2, the vibration properties of the flexible linkage, that is, mode shapes and natural frequencies, are identified by using an impact hammer (PCB 086B02), an accelerometer (PCB 333B32), and a dynamic response analyzer (Agilent 35670A). Based on the dynamic response analyzer, the first two mode shapes are validated, as illustrated in Figure 3. The first natural frequency is $92.5 \mathrm{~Hz}$ with a damping ratio of 0.041 , and the second frequency is $241.3 \mathrm{~Hz}$ with a damping ratio of 0.013 . Figure 3 shows that the estimated mode shapes match well with the pinned-free mode shapes, but there still exists some difference at the end of the linkage. The reason is that the elastic motions of the three flexible links are coupled together through the moving platform. When one flexible link suffers an impact and starts to vibrate, the other two flexible links are also forced to vibrate due to the coupled dynamic characteristic and the closed-loop nature of the parallel structure. This clearly explains why the moving platform generates vibration during the operation.

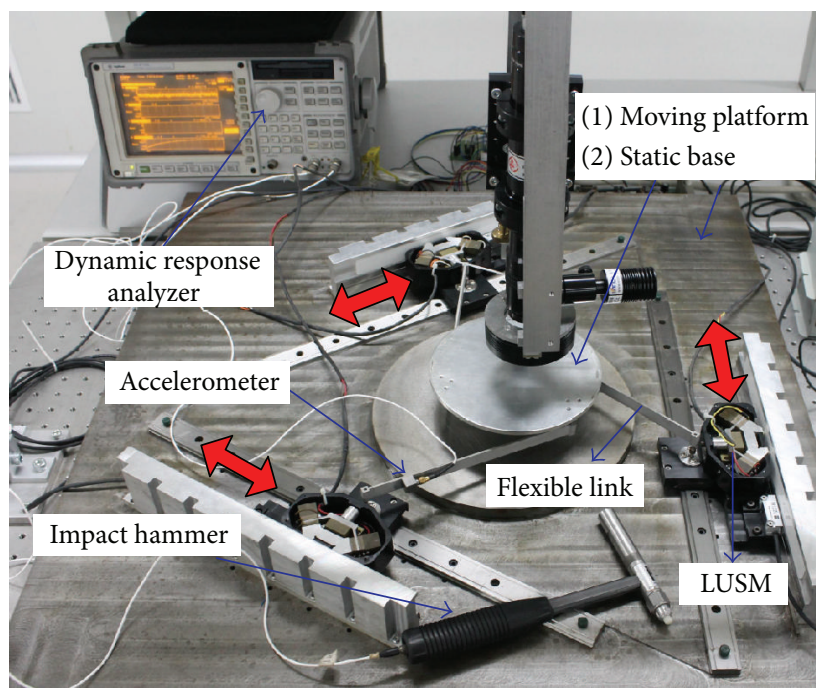

FIgURE 2: Photography of the modal test experiment setup.

The detailed analysis of the modal test experiment is shown in $[17,23]$.

2.4. Dynamic Equations of the Flexible Links. Since we focus on the vibration control of the flexible link in this study, only the dynamic equations of the elastic motion of the flexible links are formulated. For the rigid body motions, the KinetoElasto dynamics (KED) assumptions are employed to provide a prescribed rigid motion. 


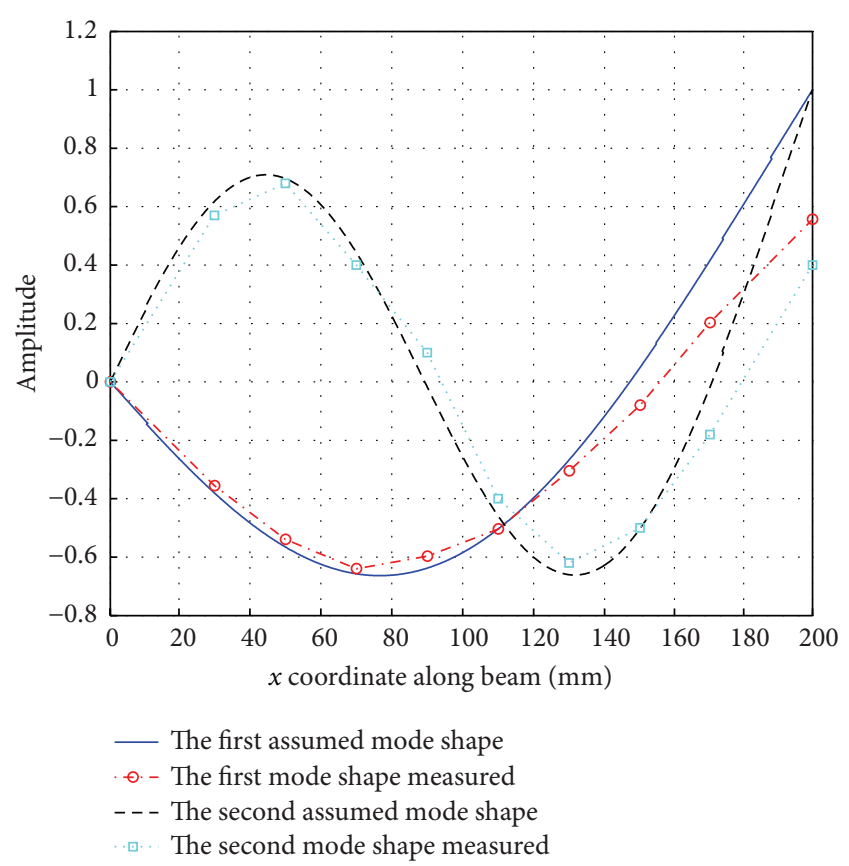

FIGURE 3: First two mode shapes of the flexible link identified.

The total kinetic energy of the PPM is presented as

$$
\begin{aligned}
T= & \sum_{i=1}^{3} \frac{1}{2} \int_{0}^{L_{i}} \rho_{A i} \dot{\bar{r}}_{i}^{2} d x+\sum_{i=1}^{3} \frac{1}{2} m_{S i} \dot{\rho}_{i}^{2} \\
& +\frac{1}{2} m_{p}\left(\dot{x}_{p}^{2}+\dot{y}_{p}^{2}\right)+\frac{1}{2} I_{p} \dot{\varphi}_{p}^{2}, \\
\bar{r}_{i}= & {\left[x_{a i}+\rho_{i} \cos \alpha_{i}+x \cos \beta_{i}-w_{i}(x, t) \sin \beta_{i}\right] i } \\
& +\left[y_{a i}+\rho_{i} \sin \alpha_{i}+x \sin \beta_{i}+w_{i}(x, t) \cos \beta_{i}\right] j,
\end{aligned}
$$

where the first and the second items of (3) represent the kinetic energies of the flexible links and the sliders, respectively, and the last two items are the kinetic energy of the moving platform. Equation (4) is defined as the position vector of $x_{i}$ on the $i$ th flexible links. Variable $m_{S i}$ is the mass of the sliders, $m_{p}$ is the mass of the moving platform, $I_{p}$ is the mass moment of the platform, $\rho_{A i}$ is the mass per unit length of the $i$ th sliders, and $\left(x_{a i}, y_{a i}\right)$ are the coordinates of point $A_{i}$ in the static frame.

Since the manipulator is moving in the $x-y$ plane, the potential energy changes because gravity is ignored, and hence only the potential energy caused by the elastic motion of the flexible links is considered. The total potential energies of the PPM are presented as

$$
V=\sum_{i=1}^{3} \frac{1}{2} \int_{0}^{L i} E_{i} I_{i}\left(w_{i}^{\prime \prime}\right)^{2} d x,
$$

where $E_{i}$ and $I_{i}$ represent the Young modulus of elasticity and the second moment of area of the $i$ th flexible link, respectively.

To implement the efficient modal control for vibration control of the PPM, multiple PZT transducers are mounted on the flexible links as vibration sensors and actuators, and the control strategies are shown in Figure 4. According to $[24,25]$, the generalized modal control forces applied by PZT actuators corresponding to the modal coordinates $q_{i j}$ are given as

$$
F_{u}^{i j}=\sum_{k=1}^{m} \lambda_{a}\left[Y_{i j}^{\prime}\left(l_{2}^{k}\right)-Y_{i j}^{\prime}\left(l_{1}^{k}\right)\right] V_{a k}^{i}(t)
$$

where $\lambda_{a}$ is the constant of PZT actuator, $l_{2}^{k}$ and $l_{1}^{k}$ are the left and right end positions of the $k$ th PZT actuator respectively, and $V_{a k}^{i}(t)$ represents the control voltages imposed on the $k$ th PZT actuator of the $i$ th flexible link.

The general form of Lagrange's equations for elastic generalized coordinates is given as

$$
\frac{d}{d t}\left(\frac{\partial(T-V)}{\partial \dot{q}_{i j}}\right)-\frac{\partial(T-V)}{\partial q_{i j}}=F_{u}^{i j},
$$

where $i=1,2,3$ and $j=1,2, \ldots, r$.

Then substituting (3)-(6) into (7) and writing the results in matrix form with considering $i=1,2,3$ yields the following equations:

$$
M \ddot{\bar{q}}+K \bar{q}=\bar{F}_{r}+\bar{F}_{u}
$$

where $\bar{q}=\left[\begin{array}{lllllllll}q_{11} & \cdots & q_{1 r} & q_{21} & \cdots & q_{2 r} & q_{31} & \cdots & q_{3 r}\end{array}\right]^{T}, \bar{F}_{u}=$ $\left[\begin{array}{lllllllll}F_{u}^{11} & \cdots & F_{q}^{1 r} & F_{u}^{21} & \cdots & F_{u}^{2 r} & F_{u}^{31} & \cdots & F_{u}^{3 r}\end{array}\right]^{T}, \bar{F}_{r}$ is the excitation modal forces arising from the rigid body motion and coupling effect between elastic and rigid motion, and $M$ and $K$ are the positive mass matrix and stiffness matrix of the three flexible links, respectively. The detailed expressions of $M, K$, and $\bar{F}_{r}$ are given in the appendix.

\section{Efficient Modal Control}

3.1. Independent Modal Space Controller. Since the EMC is based on the IMSC, the IMSC is analyzed in this chapter first. Independent modal space control has the ability to control each independent mode instead of controlling the continuous structures, with no coupling among the targeted modes. According to the dynamic equation (8), the independent modal equation for the $j$ th mode of the $i$ th flexible link is given as

$$
\ddot{q}_{i j}+\omega_{i j}^{2} q_{i j}=\frac{\left(F_{r}^{i j}+F_{u}^{i j}\right)}{m_{i j}}=f_{r}^{i j}+f_{u}^{i j},
$$

where $\omega_{i j}$ reflects the modal frequency for the $j$ th mode of the $i$ th flexible link and $m_{i j}=\int_{0}^{L_{i}} \rho_{A i} Y_{i j}^{2} d x$ is a positive constant.

With IMSC, the modal control force $f_{u}^{i j}$ is designed to only depend on the modal displacement and modal velocity as

$$
f_{u}^{i j}=-k_{p}^{i j} q_{i j}-k_{d}^{i j} \dot{q}_{i j} .
$$




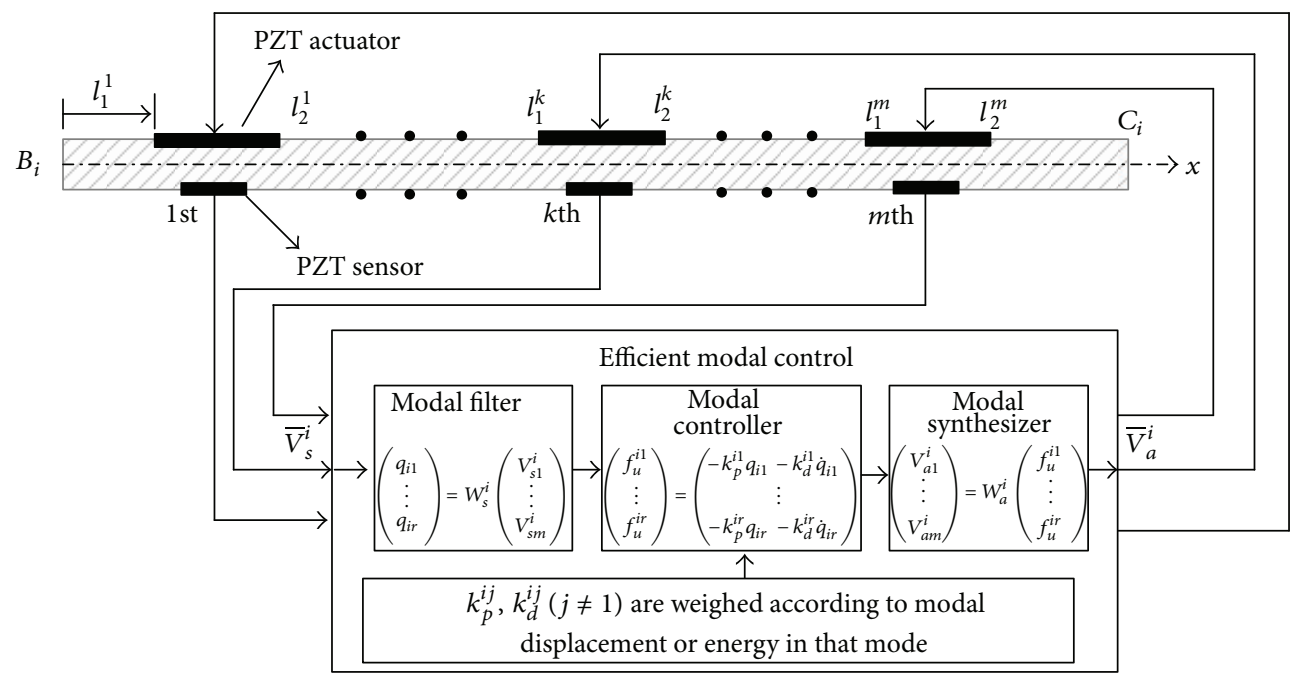

FIGURE 4: Schematic of efficient modal control.

Substituting (10) to (9) yields the closed-form equations for the $j$ th mode of the $i$ th flexible link as

$$
\ddot{q}_{i j}+k_{d}^{i j} \dot{q}_{i j}+\left(\omega_{i j}^{2}+k_{p}^{i j}\right) q_{i j}=f_{r}^{i j} .
$$

Equation (11) clearly indicates that the active damping forces and the positive stiffness forces are imported to the modal equation through the vibration actuator, thus increasing the damping and stiffness features of the flexible links. The initial values of feedback gains of $k_{p}^{i j}$ and $k_{d}^{i j}$ can be determined using either pole assignment or optimal control method. According to the optimal control approach [26], the cost function which is related to the potential energy $\omega_{i j}^{2} q_{i j}^{2}$, kinetic energy $\dot{q}_{i j}^{2}$, and the required control input $\left(f_{u}^{i j}\right)^{2}$ is selected as

$$
J=\int_{0}^{\infty}\left[\left(\omega_{i j}^{2} q_{i j}^{2}+\dot{q}_{i j}^{2}\right)+\lambda\left(f_{u}^{i j}\right)^{2}\right] d t
$$

where weighting factor $\lambda$ represent a compromise between the required control input and vibration control efficiency.

Based on [27], the solution of (12) is expressed as

$$
\begin{aligned}
& k_{p}^{i j}=\left(\omega_{i j}^{2}+\lambda^{-1}\right)^{1 / 2}-\omega_{i j}, \\
& k_{d}^{i j}=\left(2 \omega_{i j}\left(\left(\omega_{i j}^{2}+\lambda^{-1}\right)^{1 / 2}-\omega_{i j}\right)+\lambda^{-1}\right)^{1 / 2} .
\end{aligned}
$$

3.2. Efficient Modal Control. Since the control gains for higher vibration modes are much larger than the lower modes in the IMSC method, the applied control voltages will attain high values if the feedback gains are used directly without modifying. The goal of the proposed controller is to attenuate the multimode vibration simultaneously with relatively small control force. In EMC method [21], the feedback gains for each mode are modified according to its own modal displacement or energy, and hence the higher modes with lower vibration amplitude can be suppressed with low damping applied. According to EMC, only the feedback gains for the first mode are obtained through optimal control method, but the others are optimized using energy weighting method as follows:

$$
k_{p}^{i 1}: k_{p}^{i j}: k_{p}^{i r}=1: \frac{E_{i j}}{E_{i 1}} \times \frac{\omega_{i 1}}{\omega_{i j}}: \frac{E_{i r}}{E_{i 1}} \times \frac{\omega_{i 1}}{\omega_{i r}},
$$

where $E_{i j}=\omega_{i j}^{2} q_{i j}^{2}+\dot{q}_{i j}^{2}$ reflects the modal vibration energy for the $j$ th mode of the $i$ th link and $r$ represents the number of modes to be controlled.

3.3. Modal Filter and Synthesizer. Real-time monitoring of modal coordinates plays a significant role in design of modal feedback controller. As any modal feedback controller, the modal displacements and velocities are required to be extracted from the vibration sensors in the proposed method. The most common methods used to measure the modal coordinates include state observers, temporal filters, and modal filters. Compared with state observers and temporal filters, modal filters extract modal coordinates from vibration sensors independent of the control work, and hence it can be directly applied to any modal feedback controller. Furthermore, modal filters also have the advantage of preventing observation spillover from the residual modes. Therefore, modal filters with discrete PZT sensors are adopted to provide modal coordinates separation.

According to $[17,25]$, the resultant voltage generated in the PZT sensor is expressed with respect to the charge as

$$
V_{s}^{k}(t)=\frac{b E_{p} d_{31} t_{e q}}{C_{p}^{l}}\left(\frac{\partial w\left(l_{2}^{k}, t\right)}{\partial x}-\frac{\partial w\left(l_{1}^{k}, t\right)}{\partial x}\right),
$$

where $E_{p}$ is the Young modulus of PZT sensor, $d_{31}$ represents the constant of PZT materials, $b$ is the width of the PZT sensor, $C_{p}^{l}$ is the equivalent constant of piezoelectric capacitance, $t_{e q}$ is defined as the distance between neutral axis of beam/sensor and that of midplane of beam and sensor, and 
$l_{2}^{k}$ and $l_{1}^{k}$ are the left and right end positions of the $k$ th PZT sensor, respectively.

Therefore, the modal filter expression for $i$ th smart link with $m$ PZT sensor is given as [28]

$$
\bar{q}_{i}(t)=W_{s}^{i} \bar{V}_{s}^{i}(t)
$$

where $\bar{q}_{i}(t)=\left[\begin{array}{llll}q_{i 1}(t) & q_{i 2}(t) & \cdots & q_{i r}(t)\end{array}\right]^{T}$ is the modal displacement vector of the $i$ th smart link, $\bar{V}_{s}^{i}(t)=$ $\left[\begin{array}{llll}V_{s 1}^{i}(t) & V_{s 2}^{i}(t) & \cdots & V_{s m}^{i}(t)\end{array}\right]^{T}$ is the output voltage vector of the $m$ PZT sensors attached on the $i$ th smart link, and $W_{s}^{i}$ is a $r \times m$ transformation matrix expressed as

$$
W_{s}^{i}=\frac{1}{\lambda_{s}} A^{T}\left(A A^{T}\right)^{-1}
$$

where $\lambda_{s}=b E_{p} d_{31} t_{e q} / C_{p}^{l}$ is PZT sensor constant, and the matrix $A$ related to the mode shape function of the $i$ th smart link is written as

$$
\begin{aligned}
& A \\
& =\left[\begin{array}{cccc}
Y_{i 1}^{\prime}\left(l_{2}^{1}\right)-Y_{i 1}^{\prime}\left(l_{1}^{1}\right) & Y_{i 2}^{\prime}\left(l_{2}^{1}\right)-Y_{i 2}^{\prime}\left(l_{1}^{1}\right) & \cdots & Y_{i r}^{\prime}\left(l_{2}^{1}\right)-Y_{i r}^{\prime}\left(l_{1}^{1}\right) \\
Y_{i 1}^{\prime}\left(l_{2}^{2}\right)-Y_{i 1}^{\prime}\left(l_{1}^{2}\right) & Y_{i 2}^{\prime}\left(l_{2}^{2}\right)-Y_{i 2}^{\prime}\left(l_{1}^{2}\right) & \cdots & Y_{i r}^{\prime}\left(l_{2}^{2}\right)-Y_{i r}^{\prime}\left(l_{1}^{2}\right) \\
\vdots & \vdots & \vdots & \vdots \\
Y_{i 1}^{\prime}\left(l_{2}^{m}\right)-Y_{i 1}^{\prime}\left(l_{1}^{m}\right) & Y_{i 2}^{\prime}\left(l_{2}^{m}\right)-Y_{i 2}^{\prime}\left(l_{1}^{m}\right) & \cdots & Y_{i r}^{\prime}\left(l_{2}^{m}\right)-Y_{i r}^{\prime}\left(l_{1}^{m}\right)
\end{array}\right] .
\end{aligned}
$$

During the efficient modal control process, the output voltages of the PZT sensors are first transferred from physical coordinates to modal coordinates through modal filter, and then the modal coordinates are provided to the EMC controller to calculate the active modal forces. At last, the computed modal forces are converted to the control voltages corresponding to the PZT actuators in physical space using modal synthesizer. The overall control process is illustrated in Figure 4, and the modal synthesizer for $i$ th link is expressed as

$$
\bar{V}_{a}^{i}(t)=W_{a}^{i} \bar{f}_{u i}(t)
$$

where $\bar{f}_{u i}(t)=\left[\begin{array}{llll}f_{u}^{i 1}(t) & f_{u}^{i 2}(t) & \cdots & f_{u}^{i r}(t)\end{array}\right]^{T}$ is the modal control force vector calculated from EMC method for the $i$ th smart link, $\bar{V}_{a}^{i}(t)=\left[\begin{array}{llll}V_{a 1}^{i}(t) & V_{a 2}^{i}(t) & \cdots & V_{a m}^{i}(t)\end{array}\right]^{T}$ is the control voltage vector applied to the $m$ PZT actuators, and $W_{a}^{i}$ is a $m \times r$ transformation matrix expressed as

$$
W_{a}^{i}=\frac{1}{\lambda_{a}} A\left(A^{T} A\right)^{-1},
$$

where $\lambda_{a}$ is the constant of PZT actuator.

A problem that must be mentioned is about the transformation matrices $W_{s}^{i}$ and $W_{a}^{i}$. Taking the matrix $W_{s}^{i}$ used in the modal filter, for example, if the number of vibration sensors used to extract modal coordinate is as many as the vibration modes, namely, $m=r$ in (18), the matrix $W_{s}^{i}$ would be square and the extracted modal coordinates would be equal to the ideal modal coordinate. However, the vibration
TABLE 1: Parameters of PPM and PZT transducers.

\begin{tabular}{lcc}
\hline Symbol & Terms & Values \\
\hline$m_{p}$ & Mass of moving platform & $0.25 \mathrm{~kg}$ \\
$L_{i}$ & Length of the link & $200 \mathrm{~mm}$ \\
$m_{b}$ & Mass of the link & $0.011 \mathrm{~kg}$ \\
$m_{s}$ & Mass of the sliders & $0.15 \mathrm{~kg}$ \\
$\alpha_{i}$ & Angle of linear guide & $120^{\circ}, 240^{\circ}, 0^{\circ}$ \\
$E_{p}$ & PZT Young's modulus & $6.6 \times 10^{10} \mathrm{~N} / \mathrm{m}^{2}$ \\
$d_{31}$ & PZT piezoelectric constant & $-180 \times 10^{-12} \mathrm{C} / \mathrm{N}$ \\
$t_{b}$ & Beam thickness & $2 \mathrm{~mm}$ \\
$t_{p}$ & PZT transducers thickness & $0.2 \mathrm{~mm}$ \\
\hline
\end{tabular}

modes of the flexible structure are infinite, and only a limited number of sensors can be mounted on the flexible structure. Hence, the modal coordinates extracted from modal filter are only an approximation of the ideal modal coordinates, and the accuracy of the approximation is related to the number of the vibration sensors. In practice, increasing the number of the vibration sensors will put computational burden and make the system more complicated. The same problem also exists in the matrix $W_{a}^{i}$ of the modal synthesizer. Therefore, a compromise between real-time computing capability and the number of vibration transducers must be made when designing the overall system.

3.4. Simulation Results. Numerical simulations in MATLAB software are performed first to verify the proposed control method. During the simulation, a circular trajectory motion of the moving platform is selected as the rigid motion input, as illustrated in Figure 1. The circular motion is given as

$$
\begin{aligned}
& x_{p}=10 \cos (10 \pi t)-10(\mathrm{~mm}), \\
& y_{p}=10 \sin (10 \pi t)(\mathrm{mm}) \\
& \varphi_{p}=\frac{\pi}{6}(\mathrm{rad}) .
\end{aligned}
$$

The distance among points $A_{i}$ and $C_{i}$ is $600 \mathrm{~mm}$ and $120 \mathrm{~mm}$, respectively. The effective stroke of each LUSM is $250 \mathrm{~mm}$. Three pairs of PZT transducers are mounted at the locations of $50 \mathrm{~mm}, 100 \mathrm{~mm}$, and $150 \mathrm{~mm}$ from $B_{i}$ on the flexible link as vibration sensors and actuators. The other parameters of the system are detailed in Table 1 . The first three modes of the flexible links are selected to be controlled in the simulation work. Using the energy weighting method, the modified ratio for the second and third modes of the three flexible links are derived from (14) as

$$
\begin{aligned}
& k_{p}^{11}: k_{p}^{12}: k_{p}^{13}=k_{d}^{11}: k_{d}^{12}: k_{d}^{13}=1: 0.315: 0.041 \\
& k_{p}^{21}: k_{p}^{22}: k_{p}^{23}=k_{d}^{21}: k_{d}^{22}: k_{d}^{23}=1: 0.384: 0.036 \\
& k_{p}^{31}: k_{p}^{32}: k_{p}^{33}=k_{d}^{31}: k_{d}^{32}: k_{d}^{33}=1: 0.291: 0.044
\end{aligned}
$$

Due to the different driving forces applied by LUSM, the vibration responses of the three flexible links are different. 

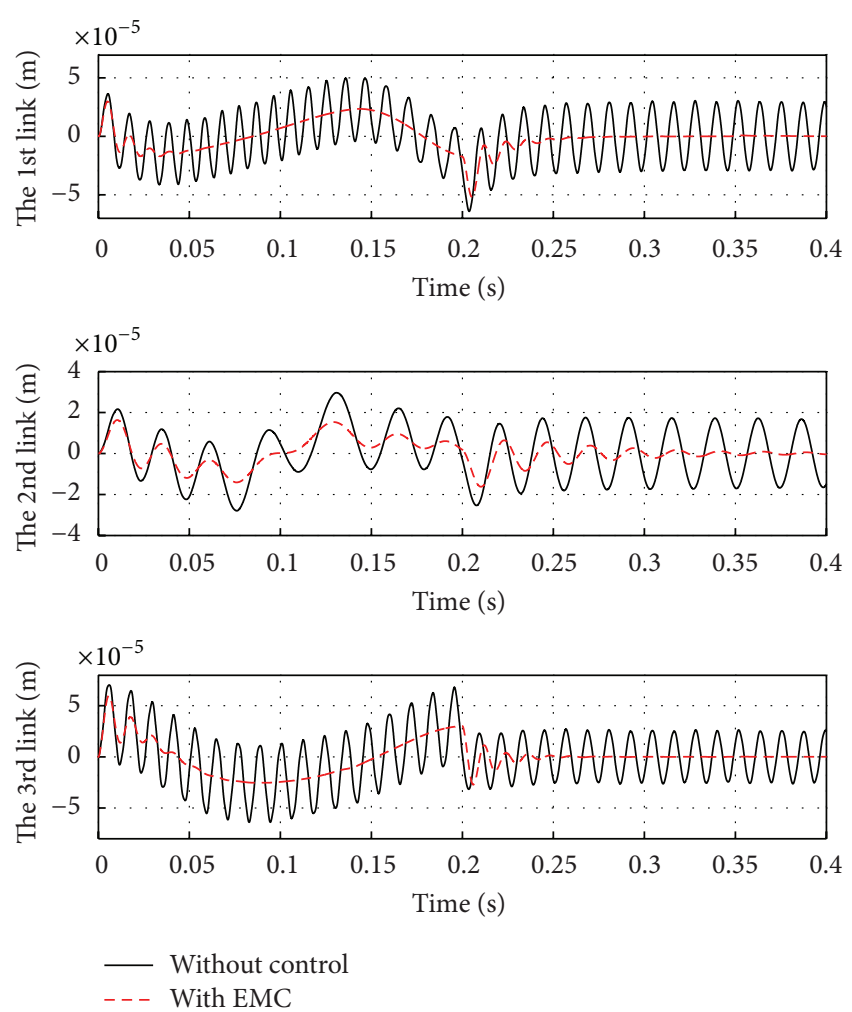

FIGURE 5: The vibration responses at the three quarters of the three flexible links.

Since the modified ratios are based on the uncontrolled system response, it can be observed in (22) that the feedback ratios for the three flexible links are not identical. The vibration responses of the three flexible links are shown in Figure 5, which reveal that the oscillations of the three flexible links are suppressed rapidly with the proposed EMC strategy. Figure 6 shows that the first three modes' vibrations of the first flexible link are all attenuated effectively, which further validate that the EMC method can suppress multimode vibration simultaneously.

\section{Experimental Results}

The trajectory of the moving platform in the experiment is the same as that used in the numerical simulation. To guarantee the accuracy of the desired motion, the kinematic calibration work of the flexible PPM is carried out based on the visual feedback and the particle swarm optimization (PSO) algorithm. The motion control card (DMC-1842, Galil) is used to control the three LUSMs through three LUSM drivers (made by NUAA). The feedback signal is provided by the linear grating sensor (LIA20, NUMERIK JENA). A DSP control board (Seed_DEC2812) is adopted to realize the active vibration control. PZT power amplifiers (XE-501, XMT) are adopted to amplify the control voltage of the PZT actuators. In the experiment, only the first flexible link is targeted to be controlled due to the limitation of the hardware. Three pairs of PZT sensors and actuators (PZT5, CSSC) are mounted
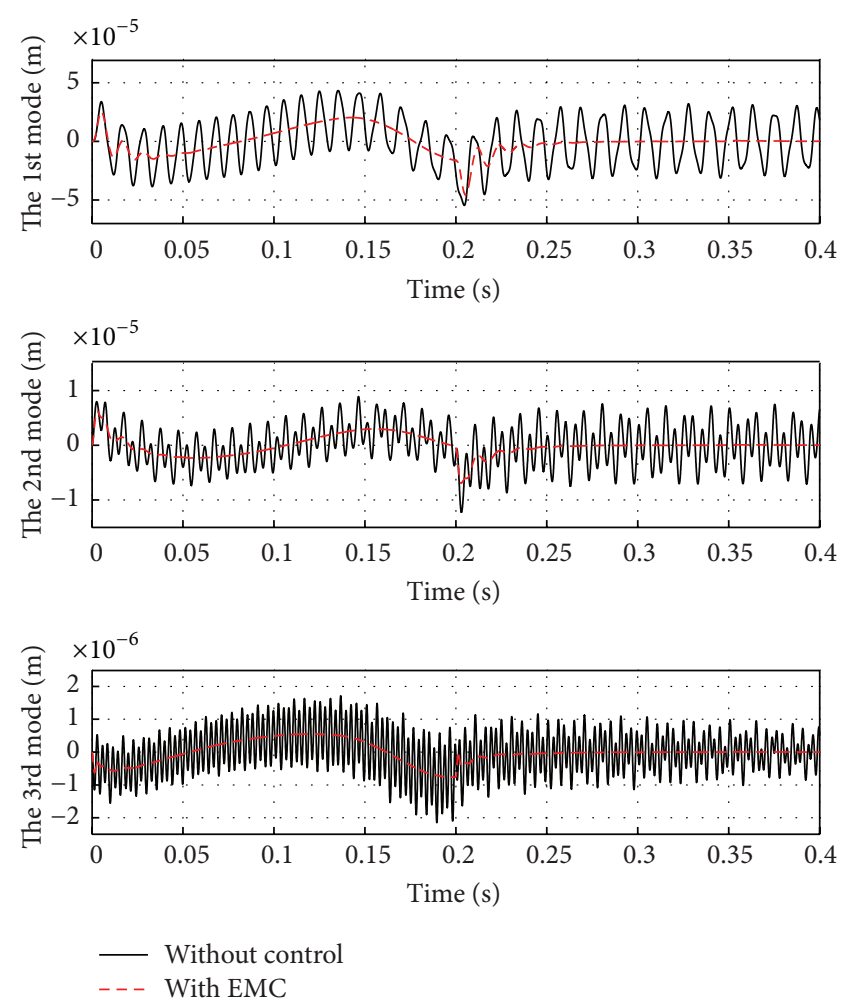

FIgURE 6: The first three mode vibrations at the three quarters of the first flexible link.

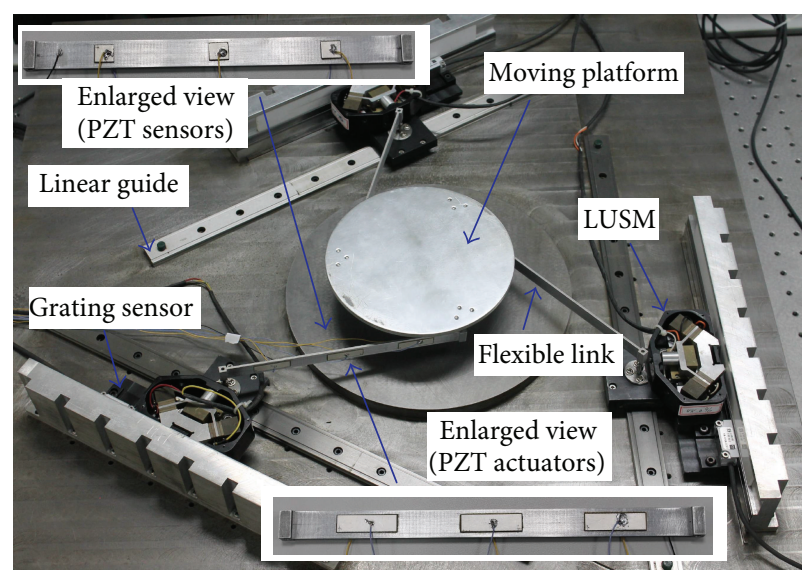

Figure 7: Arrangement of the PZT transducers.

on the location of quarter, midpoint, and three quarters of the first flexible link, as shown in Figure 7. The sizes of each sensor are $10 \times 10 \times 0.2 \mathrm{~mm}$ and the sizes of each actuator are $30 \times 10 \times 0.2 \mathrm{~mm}$.

Experiments with and without EMC method are carried out to validate the effectiveness of the proposed approach. Before the experiment, the uncontrolled systems are analyzed first to provide the required information such as modal displacements and velocities. The first two modes of the first flexible link are chosen to be suppressed in the experiment. Based on the proposed EMC approach, the feedback gains for 


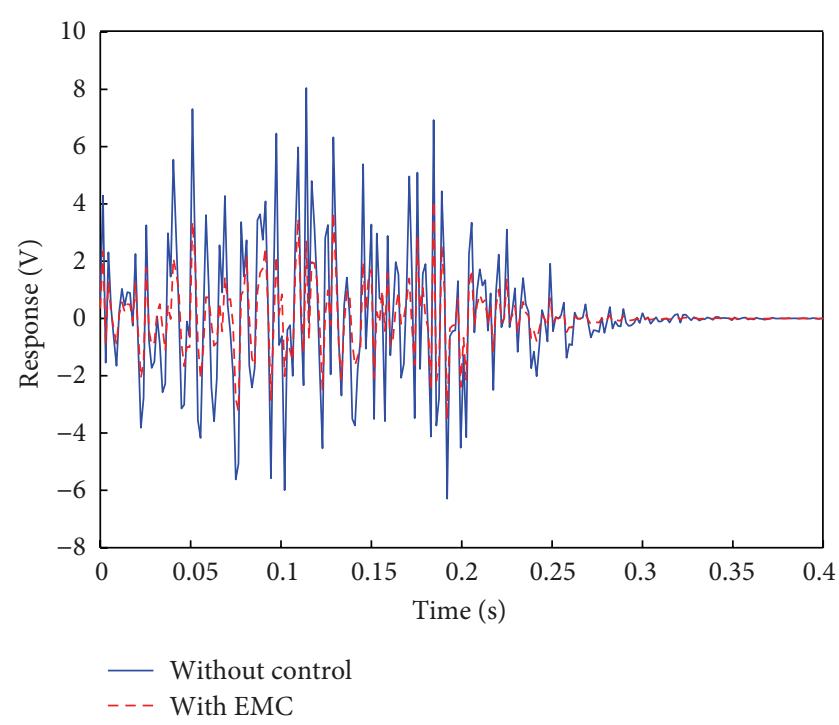

FIGURE 8: Vibration response at the quarter point of the first link.

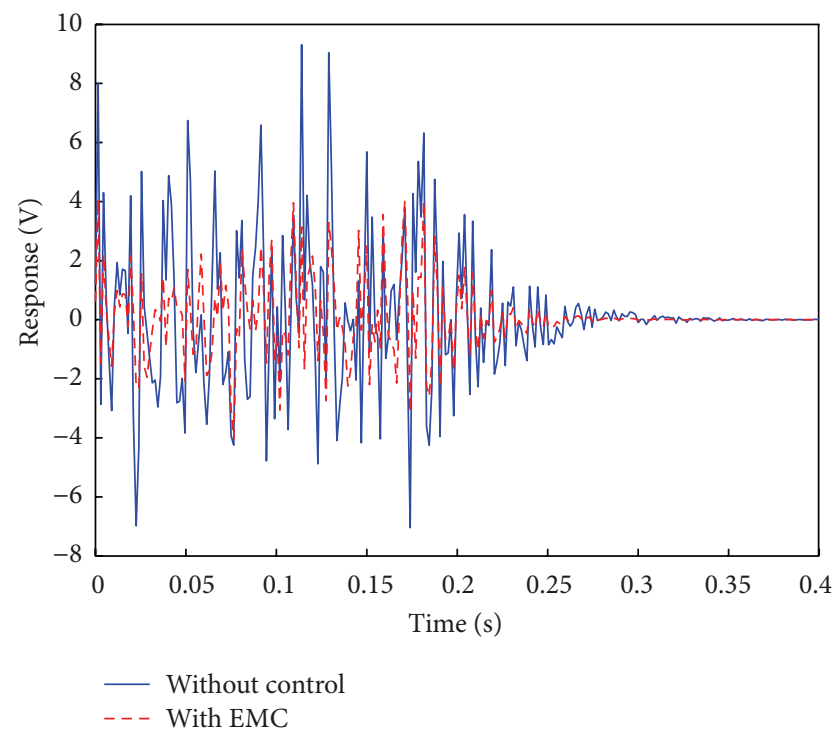

FIGURE 9: Vibration response at the midpoint of the first link.

the first mode of the first link are calculated as $k_{p}^{11}=118.7$ and $k_{d}^{11}=24.3$, and the ratio for the second mode is optimized as $k_{p}^{11}: k_{p}^{12}=k_{d}^{11}: k_{d}^{12}=1: 0.259$. The response voltages of the three PZT sensors mounted on the first flexible link are illustrated in Figures 8, 9, and 10, which clearly show that both the structural vibrations and the residual vibration of the first link are suppressed effectively with the EMC method. Furthermore, Figures 8-10 also demonstrate that the residual vibrations can be completely attenuated within $0.08 \mathrm{~s}$ after motion stops. Based on the fast Fourier transform (FFT), the first two vibration modes extracted from the PZT sensors are analyzed. Figures 11-12 show that the vibration amplitudes for the first two vibration modes at $92 \mathrm{~Hz}$ and $244 \mathrm{~Hz}$ are reduced by $57.78 \%$ and $44.96 \%$, respectively. These PSD plots

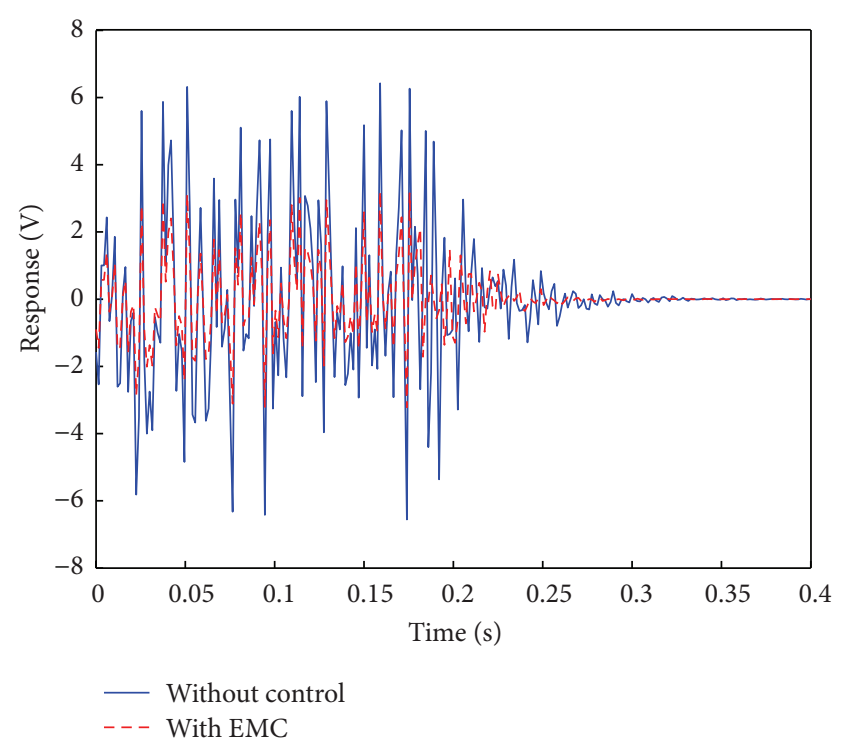

FIgURE 10: Vibration response at the three-quarter point of the first link.

verify that the proposed EMC method has the capability of suppressing multimode vibration simultaneously.

From Figures 11-12, we also find that there still exist other frequencies besides the dominant natural frequencies. In fact, as mentioned in Section 3.3, if the vibration sensors are as many as vibration modes, then the modal coordinates can be extracted completely from the other frequencies, and the other vibration frequencies will not be observed in Figures 11-12. However, the vibration modes are infinite and usually the hardware is limited in practice (in our study only three PZT sensors are adopted). Hence the number of vibration modes which can be sensed are limited and the residual mode may participate in the extracted vibration mode; for example, the first natural frequency of $92 \mathrm{~Hz}$ is observed in Figure 12 and the second natural frequency of $244 \mathrm{~Hz}$ is observed in Figure 11. Besides, many other forced vibration components are also shown in Figures 11-12, such as $33 \mathrm{~Hz}, 64 \mathrm{~Hz}$, and $144 \mathrm{~Hz}$. These forced vibration frequencies are mainly from the movement of the sliders and flexible links, the dynamics of the LUSM, and the coupling effect between the rigid body motion and elastic motion. Since these forced vibration frequencies are usually away from the natural frequencies, the active damping force has nearly no effect on these forced vibration. Hence it is shown in Figures 11-12 that the amplitudes of these forced vibrations are almost unchanged during the control experiment. For further suppressing these forced vibrations, the joint motion control methods, such as singular perturbation approach or nonlinear control method, may be adopted to optimize the driving forces of the three LUSM.

\section{Conclusions}

This paper addresses the dynamic modeling of a PPM with flexible linkage driven by LUSMs. The Lagrange equations 


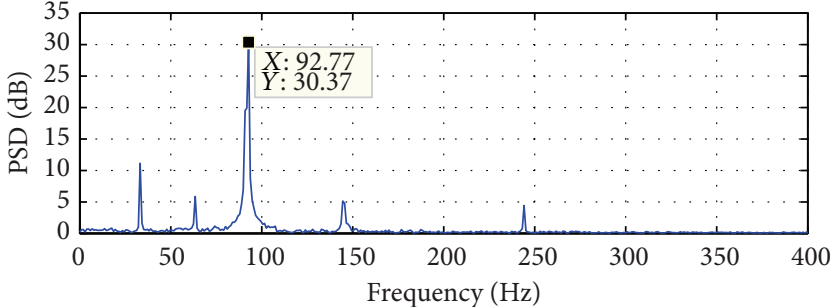

Without control

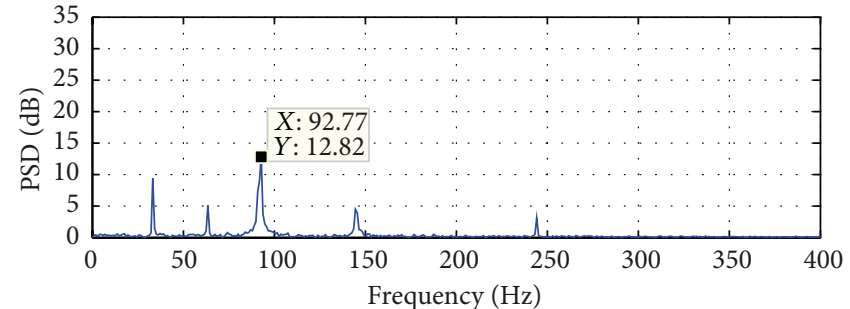

With EMC

(a)

(b)

FIGURE 11: FFT of the first vibration mode of the first link.

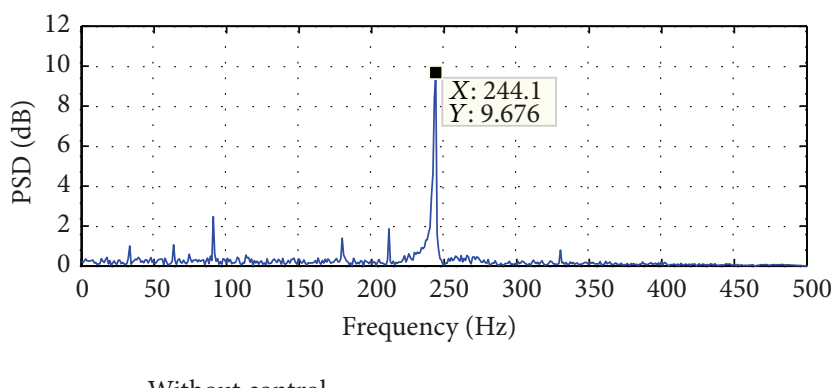

(a)

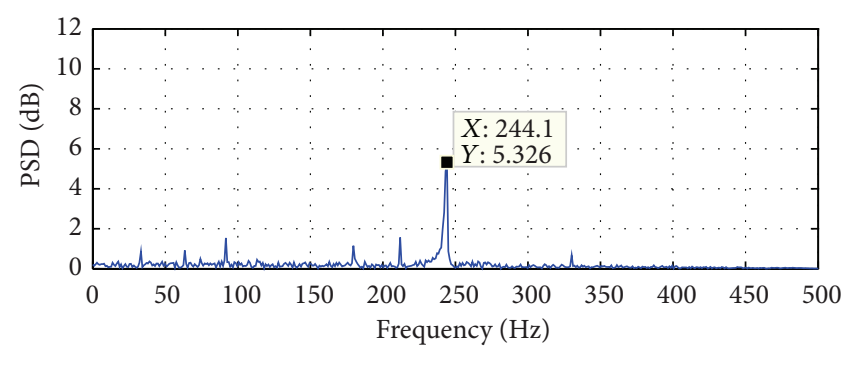

— With EMC

(b)

FIGURE 12: FFT of the second vibration mode of the first link.

and the AMM method are adopted to deduce the dynamic equations of the flexible link. With multiple PZT transducers mounted on the flexible links as vibration sensors and actuators, the EMC method is designed to suppress the unwanted vibration of the flexible link. Computer simulation and experiments are both implemented, and the results show that both the structural and residual vibrations of the flexible links are effectively suppressed when using EMC approach. Furthermore, the multimode vibration control capability of the proposed EMC method is also validated through FFT analysis. In the near future, the vibration control through joint motion controller may be employed to combine with the EMC approach to achieve further vibration suppression of the flexible link.

\section{Appendix}

Consider

$$
\begin{gathered}
M=\left[\begin{array}{ccc}
M^{1} & 0 & 0 \\
0 & M^{2} & 0 \\
0 & 0 & M^{3}
\end{array}\right] \in R^{3 r \times 3 r}, \\
M^{i}=\left[\begin{array}{ccc}
\int_{0}^{L_{i}} \rho_{A i} Y_{i 1}^{2} d x & \cdots & 0 \\
\vdots & \cdots & \vdots \\
0 & \cdots & \int_{0}^{L_{i}} \rho_{A i} Y_{i r}^{2} d x
\end{array}\right] \in R^{r \times r},
\end{gathered}
$$

$\bar{F}_{r}$

$$
\left(\begin{array}{c}
\ddot{\rho}_{1} S_{1} \int_{0}^{L_{1}} \rho_{A 1} Y_{11} d x+\ddot{\beta}_{1} \int_{0}^{L_{1}} \rho_{A 1} x Y_{11} d x-\dot{\beta}_{1}^{2} q_{11} \int_{0}^{L_{1}} \rho_{A 1} Y_{11}^{2} d x \\
\vdots \\
\ddot{\rho}_{1} S_{1} \int_{0}^{L_{1}} \rho_{A 1} Y_{1 r} d x+\ddot{\beta}_{1} \int_{0}^{L_{1}} \rho_{A 1} x Y_{1 r} d x-\dot{\beta}_{1}^{2} q_{1 r} \int_{0}^{L_{i}} \rho_{A 1} Y_{1 r}^{2} d x \\
\ddot{\rho}_{2} S_{2} \int_{0}^{L_{2}} \rho_{A 2} Y_{21} d x+\ddot{\beta}_{2} \int_{0}^{L_{2}} \rho_{A 2} x Y_{21} d x-\dot{\beta}_{2}^{2} q_{21} \int_{0}^{L_{2}} \rho_{A 2} Y_{21}^{2} d x \\
\vdots \\
\ddot{\rho}_{2} S_{2} \int_{0}^{L_{2}} \rho_{A 2} Y_{2 r} d x+\ddot{\beta}_{2} \int_{0}^{L_{2}} \rho_{A 2} x Y_{2 r} d x-\dot{\beta}_{2}^{2} q_{2 r} \int_{0}^{L_{2}} \rho_{A 2} Y_{2 r}^{2} d x \\
\ddot{\rho}_{3} S_{3} \int_{0}^{L_{3}} \rho_{A 3} Y_{31} d x+\ddot{\beta}_{3} \int_{0}^{L_{3}} \rho_{A 3} x Y_{31} d x-\dot{\beta}_{3}^{2} q_{31} \int_{0}^{L_{3}} \rho_{A 3} Y_{31}^{2} d x \\
\vdots \\
\ddot{\rho}_{3} S_{3} \int_{0}^{L_{3}} \rho_{A 3} Y_{3 r} d x+\ddot{\beta}_{3} \int_{0}^{L_{3}} \rho_{A 3} x Y_{3 r} d x-\dot{\beta}_{3}^{2} q_{3 r} \int_{0}^{L_{3}} \rho_{A 3} Y_{3 r}^{2} d x
\end{array}\right)
$$




$$
K^{i}=\left[\begin{array}{ccc}
E I \int_{0}^{L_{i}} Y_{i 1}^{\prime \prime 2} d x & \cdots & 0 \\
\vdots & \cdots & \vdots \\
0 & \cdots & E I \int_{0}^{L_{i}} Y_{i r}^{\prime \prime 2} d x
\end{array}\right] \in R^{r \times r}
$$

\section{Conflict of Interests}

The authors declare that there is no conflict of interests regarding the publication of this paper.

\section{Acknowledgments}

This work was supported by the National Natural Science Foundation of China (Grants nos. 91223201, 51375225, and 51175264), the Natural Science Research Project of Jiangsu (Grant no. BK2012797), the Open Fund of Postgraduate Innovative Base (Laboratory) for Nanjing University of Aeronautics and Astronautics (kfjj120201) and the Fundamental Research Funds for the Central Universities, and a Project Funded by the Priority Academic Program Development of Jiangsu Higher Education Institutions.

\section{References}

[1] I. Payo, V. Feliu, and O. D. Cortázar, "Force control of a very lightweight single-link flexible arm based on coupling torque feedback," Mechatronics, vol. 19, no. 3, pp. 334-347, 2009.

[2] S. Zhou and J. Shi, "Active balancing and vibration control of rotating machinery: a survey," Shock and Vibration Digest, vol. 33, no. 5, pp. 361-371, 2001.

[3] Y. Shen and A. Homaifar, "Vibration control of flexible structures with PZT sensors and actuators," JVC/Journal of Vibration and Control, vol. 7, no. 3, pp. 417-451, 2001.

[4] X. Zhang, J. K. Mills, and W. L. Cleghorn, "Multi-mode vibration control and position error analysis of parallel manipulator with multiple flexible links," Transactions of the Canadian Society for Mechanical Engineering, vol. 34, no. 2, pp. 197-213, 2010.

[5] K. Krishnamurthy and L. Yang, "Dynamic modeling and simulation of two cooperating structurally-flexible robotic manipulators," Robotica, vol. 13, no. 4, pp. 375-384, 1995.

[6] C. M. A. Vasques and J. Dias Rodrigues, "Active vibration control of smart piezoelectric beams: comparison of classical and optimal feedback control strategies," Computers and Structures, vol. 84, no. 22-23, pp. 1402-1414, 2006.

[7] Q. Hu and G. Ma, "Vibration suppression of flexible spacecraft during attitude maneuvers," Journal of Guidance, Control, and Dynamics, vol. 28, no. 2, pp. 377-380, 2005.

[8] S. K. Dwivedy and P. Eberhard, "Dynamic analysis of flexible manipulators, a literature review," Mechanism and Machine Theory, vol. 41, no. 7, pp. 749-777, 2006.

[9] X. Wang and J. K. Mills, "Dynamic modeling of a flexiblelink planar parallel platform using a substructuring approach," Mechanism and Machine Theory, vol. 41, no. 6, pp. 671-687, 2006.

[10] G. Piras, W. L. Cleghorn, and J. K. Mills, "Dynamic finiteelement analysis of a planar high-speed, high-precision parallel manipulator with flexible links," Mechanism and Machine Theory, vol. 40, no. 7, pp. 849-862, 2005.

[11] X. Zhang, J. K. Mills, and W. L. Cleghorn, "Vibration control of elastodynamic response of a 3-PRR flexible parallel manipulator using PZT transducers," Robotica, vol. 26, no. 5, pp. 655-665, 2008.

[12] Y. Yu, Z. Du, J. Yang, and Y. Li, "An experimental study on the dynamics of a 3-RRR flexible parallel robot," IEEE Transactions on Robotics, vol. 27, no. 5, pp. 992-997, 2011.

[13] J. Shan, H. T. Liu, and D. Sun, "Slewing and vibration control of a single-link flexible manipulator by positive position feedback (PPF)," Mechatronics, vol. 15, no. 4, pp. 487-503, 2005.

[14] X. Zhang, J. K. Mills, and W. L. Cleghorn, "Flexible linkage structural vibration control on a 3-PRR planar parallel manipulator: experimental results," Proceedings of the Institution of Mechanical Engineers I: Journal of Systems and Control Engineering, vol. 223, no. 1, pp. 71-84, 2009.

[15] Z. Chu, J. Cui, and F. Sun, "Vibration control of a highspeed manipulator using input shaper and positive position feedback," in Knowledge Engineering and Management, pp. 599609, Springer, Berlin, Germany, 2014.

[16] Q. Zhang, J. Mills K, L. W. Cleghorn, J. Jina, and Z. Sun, "Dynamic model and input shaping control of a flexible link parallel manipulator considering the exact boundary conditions," Robotica, 2014.

[17] Q . Zhang, K. J. Mills, L. W. Cleghorn et al., “Trajectory tracking and vibration suppression of a 3-PRR parallel manipulator with flexible links," Multibody System Dynamics, pp. 1-34, 2013.

[18] X. Zhang, Dynamic Modeling and Active Vibration Control of a Planar 3-PRR Parallel Manipulator with Three Flexible Links, University of Toronto, Toronto, Canada, 2009.

[19] L. Meirovitch and H. Baruh, "Optimal control of damped flexible gyroscopic systems," Journal of Guidance, Control, and Dynamics, vol. 4, no. 2, pp. 157-163, 1981.

[20] A. Baz and S. Poh, "Performance of an active control system with piezoelectric actuators," Journal of Sound and Vibration, vol. 126, no. 2, pp. 327-343, 1988.

[21] S. P. Singh, H. S. Pruthi, and V. P. Agarwal, "Efficient modal control strategies for active control of vibrations," Journal of Sound and Vibration, vol. 262, no. 3, pp. 563-575, 2003.

[22] L. Meirovitch, Fundamentals of Vibrations, Waveland Press, 2010.

[23] Q. Zhang, L. Zhou, J. Zhang et al., "Efficient modal control for vibration suppression of a flexible parallel manipulator," in Proceedings of the IEEE International Conference on Robotics and Biomimetics (ROBIO '13), pp. 13-18, 2013.

[24] X. Zhang, X. Wang, J. K. Mills, and W. L. Cleghorn, "Dynamic modeling and active vibration control of a 3-PRR flexible parallel manipulator with PZT transducers," in Proceedings of the 7th World Congress on Intelligent Control and Automation (WCICA '08), pp. 461-466, June 2008.

[25] N. Jalili, Piezoelectric-Based Vibration Control: From Macro to Micro/Nano Scale Systems, Springer, New York, NY, USA, 2010.

[26] F. Lin, Robust Control Design: An Optimal Control Approach, John Wiley \& Sons, 2007.

[27] L. Meirovitch, Dynamics And Control Of Structures, John Wiley \& Sons, New York, NY. USA, 1990.

[28] H. Sumali, K. Meissner, and H. H. Cudney, "A piezoelectric array for sensing vibration modal coordinates," Sensors and Actuators, A: Physical, vol. 93, no. 2, pp. 123-131, 2001. 

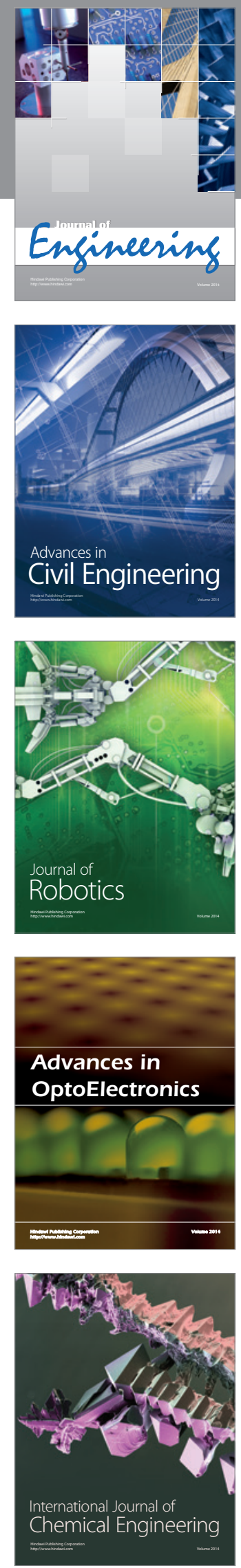

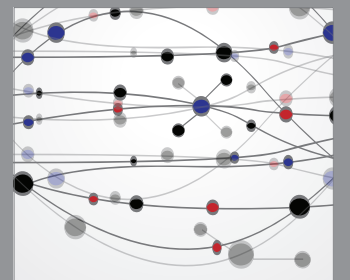

The Scientific World Journal
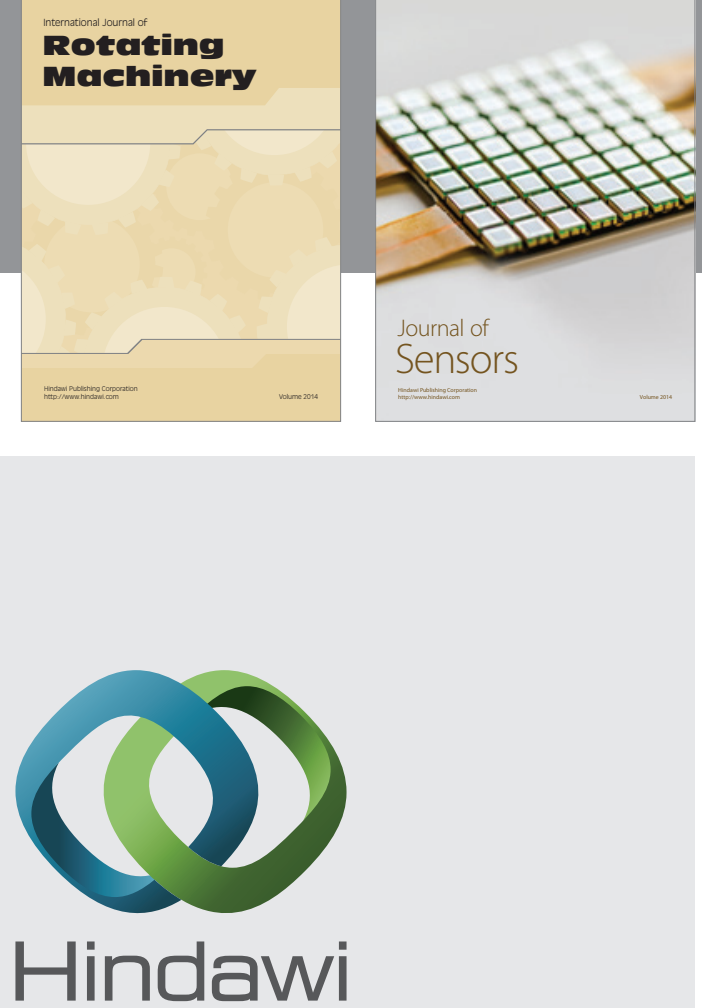

Submit your manuscripts at http://www.hindawi.com
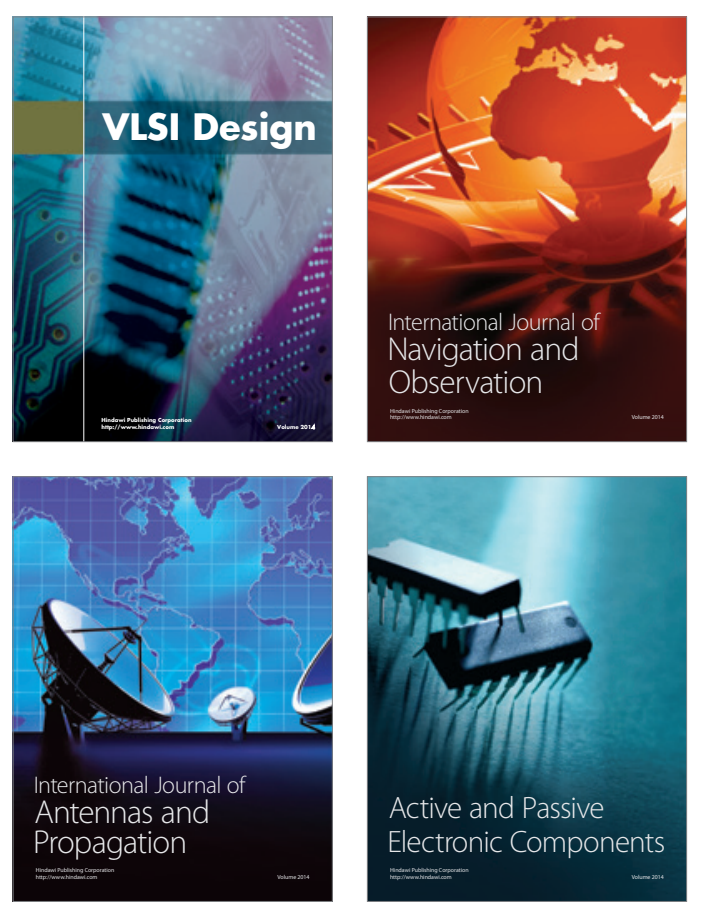
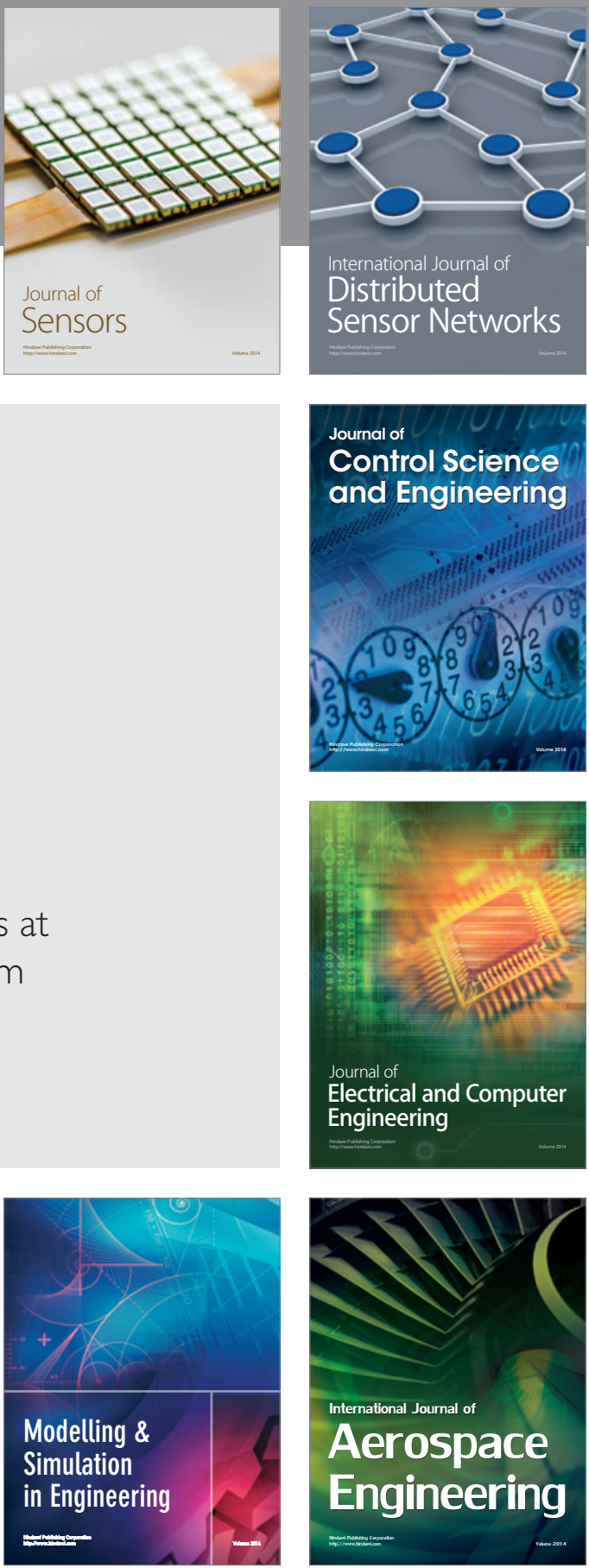

Journal of

Control Science

and Engineering
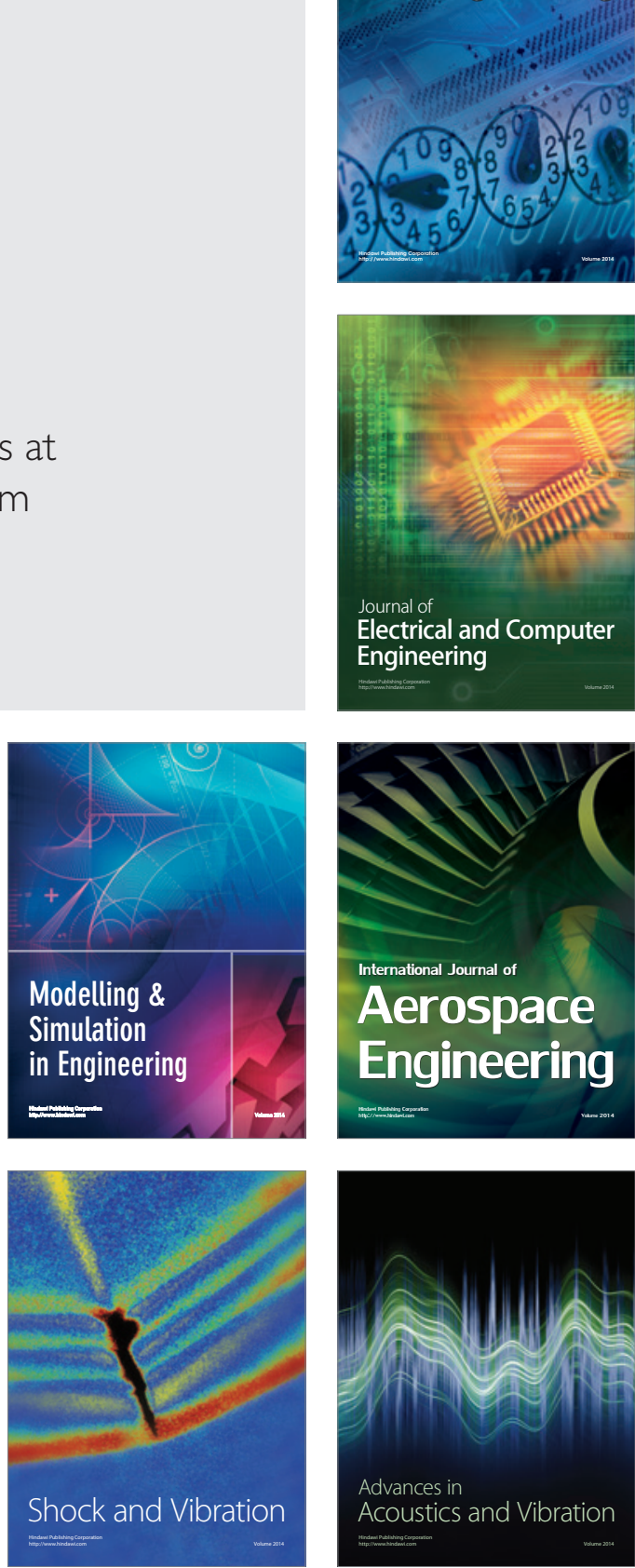